\title{
Long-Chain Acyl-CoA Dehydrogenase Deficiency
}

National Cancer Institute

\section{Source}

National Cancer Institute. Long-Chain Acyl-CoA Dehydrogenase Deficiency. NCI

Thesaurus. Code C84537.

A genetic disorder characterized by deficiency of the enzyme long-chain acyl-coenzyme A dehydrogenase that metabolizes long-chain fatty acids. Signs and symptoms appear in infancy or childhood and may be triggered during fasting, illness or exercise. They include hypoglycemia, muscle weakness and lethargy. 